\title{
Alcohols detection by a polymeric sensing film deposited on a long-period fiber grating
}

\author{
S. Muñoz-Aguirre, J. Castillo-Mixcóatl and G. Beltrán-Pérez \\ Facultad de Ciencias Físico Matemáticas, Benemérita Universidad Autónoma de Puebla, \\ Avenida San Claudio y 18 Sur, Col. San Manuel CU Puebla 72570, México.
}

Received 11 December 2020; accepted 4 February 2021

\begin{abstract}
In this paper, we report the construction and characterization of a sensor to detect volatile organic compounds, particularly alcohols. The sensor was fabricated by depositing a polydimethylsiloxane sensing film on the surface of a long-period fiber grating (LPFG). The LPFG spectrum, which was taken as the sensor response, was measured in a Teflon chamber, where different concentrations of methanol, ethanol, propanol, and butanol samples were injected. Spectral shifts to lower wavelengths were observed for the last three samples. However, a shift to higher wavelengths for the case of methanol was observed, which was attributed to the fact that its refractive index is smaller than the one for PDMS. Therefore, curves of the sensor response in function of concentration were performed using different analysis techniques: analysis in a fixed wavelength, analysis in a fixed spectrum amplitude, and spectral analysis through principal components analysis (PCA) method. The results showed a regular behavior of the responses according to the molecular weight of the samples. A discussion of the use of each technique was performed.
\end{abstract}

Keywords: Long-period fiber grating; organic vapor sensor; polydimethylsiloxane; principal components analysis.

PACS: $42.81 .-\mathrm{i} ; 42.81 . \mathrm{Pa}$

\section{Introduction}

Studies of volatile organic compound (VOC) detection has been of interest in science due to its multiple applications, such as quality control of food and beverages [1], cosmetic industry, drug or explosive detection [2], and so on. Therefore, it is important to develop devices or systems that can detect the presence of VOCs. Although there is a variety of sensors to detect such compounds [3-5], long-period fiber gratings (LPFG) have been studied since they enable remote measurements to be performed, they are immune to electromagnetic radiation and, it is possible to use them in highly noisy environments or in places where there are explosion risks. In particular, LPFGs are devices that can couple light radiation of the modes propagating in the core of an optical fiber into the modes propagating in the cladding of this fiber [6-9]. The propagation characteristics are strongly influenced by the surrounding environment properties like refractive index, hence a sensing film deposited around the fiber can be such an environment, which can provoke an increment of the sensitivity [10]. This interaction can be measured as changes in the transmission spectrum of the LPFG. The sensor selectivity can be changed by deposition around the LPFG of a sensing film that can be made of different materials, such as polydimethylsiloxane (PDMS), due to its interaction with VOC [11], some of the PDMS interesting points are its affinity to alcohols, reversible sensor response besides some characteristics as low cost, simple deposition on the fiber surface and its refractive index is lower than that of the fiber, which allows observing the interaction as spectrum shifts [12]. Currently, there are organic vapor sensors that use the principle mentioned above and special quartz optical fiber with low $\mathrm{OH}$ concentration [13]. Results for the detection of trichloroethylene, 1,1-dichloroethylene, and benzene have been reported.

On the other hand, a research paper has also reported the use of PDMS as sensing film deposited on an LPFG coupled to a ring cavity composed of fiber Bragg gratings (FBG) [14]. In this setup, sensor responses for xylene, trichloroethylene, cyclohexane, and gasoline have been obtained. The best response was obtained for xylene, whereas the other samples presented a response with significant fluctuations, especially the sample of gasoline. Furthermore, VOC detection has been reported by using different materials, such as zeolites or calixarenes as the sensing film deposited on an LPFG produced with a CO2 laser [15]. These materials present results of interaction with isopropanol and toluene at very low concentrations (49.5 ppm for isopropanol and $1.1 \mathrm{ppm}$ for toluene).

Since VOCs are important to the industry of food and beverages, and even for their applications as fuels, this paper presents an application of a PDMS sensing film deposited on the surface of an LPFG recorded on an SMF-28 optical fiber to detect methanol, ethanol, 1-propanol, and 1-butanol. The LPFG was constructed by the point-to-point method through the application of an electric arc, which is a relatively simple method to implement. The LPFG spectrum was measured with a conventional optical power meter, and processed by a PC. The results revealed increments, attenuations, and shifts in the LPFG transmission spectra, which may be related to vapor concentration. Besides a study on sensitivity related to optical power, wavelength shifts, and spectral analysis performed by principal components analysis (PCA) related to the kind of alcohol and its molecular weight was performed. 


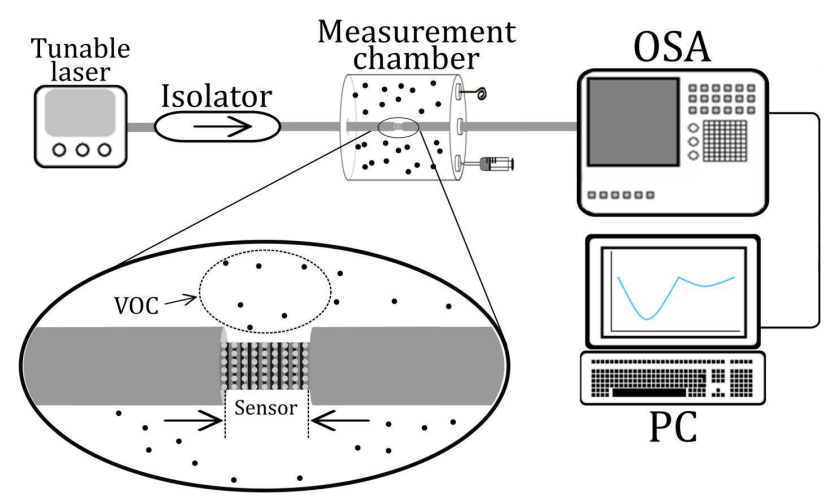

FIGURE 1. Experimental setup to measure the sensor response.

\section{Experimental setup}

The sensor was developed in three stages: (1) LPFG construction, (2) polymeric sensing film deposition, and (3) sensor response characterization at different alcohol vapor concentrations. The LPFG was constructed by the point-to-point method using electric arcs generated with a fusion splicer (S176, FITEL). Among the electric arc technique advan- tages, there are the simplicity of the writing, low fabrication cost, high thermal stability, and the possibility of writing the LPFG on any kind of fiber [16]. The arcs were applied to a portion of a standard optical fiber SMF-28 (Thorlabs, Inc.), which was previously uncovered in approximately 4 $\mathrm{cm}$ long. The fiber was fixed at one end by a linear actuator (T-LA28, ZABER Technologies, Inc.), which allowed moving the fiber to apply the electric arcs at constant distances. A 2 gr weight was placed at the other end to tighten the fiber and avoid deformations. Moreover, one fiber end was connected to a power meter (PM100, Thorlabs, Inc.), which transmitted the data to a PC to monitor the transmission spectrum of the LPFG in situ during its construction. The polymeric sensing film was deposited on the LPFG surface by the immersion technique. The sensing film material was PDMS (SigmaAldrich), previously diluted in chloroform at a concentration of $0.28 \mathrm{gr} / \mathrm{ml}$. The sensor response characterization was performed in a Teflon measurement chamber, where the liquid alcohol samples were injected.

The experimental setup, as shown in Fig. 1, consisted of a tunable laser diode (6528-HR, New Focus, Inc.), which covered a bandwidth of $100 \mathrm{~nm}$ (from 1520 to $1620 \mathrm{~nm}$ ) to measure the LPFG transmission spectrum; a Teflon measurement
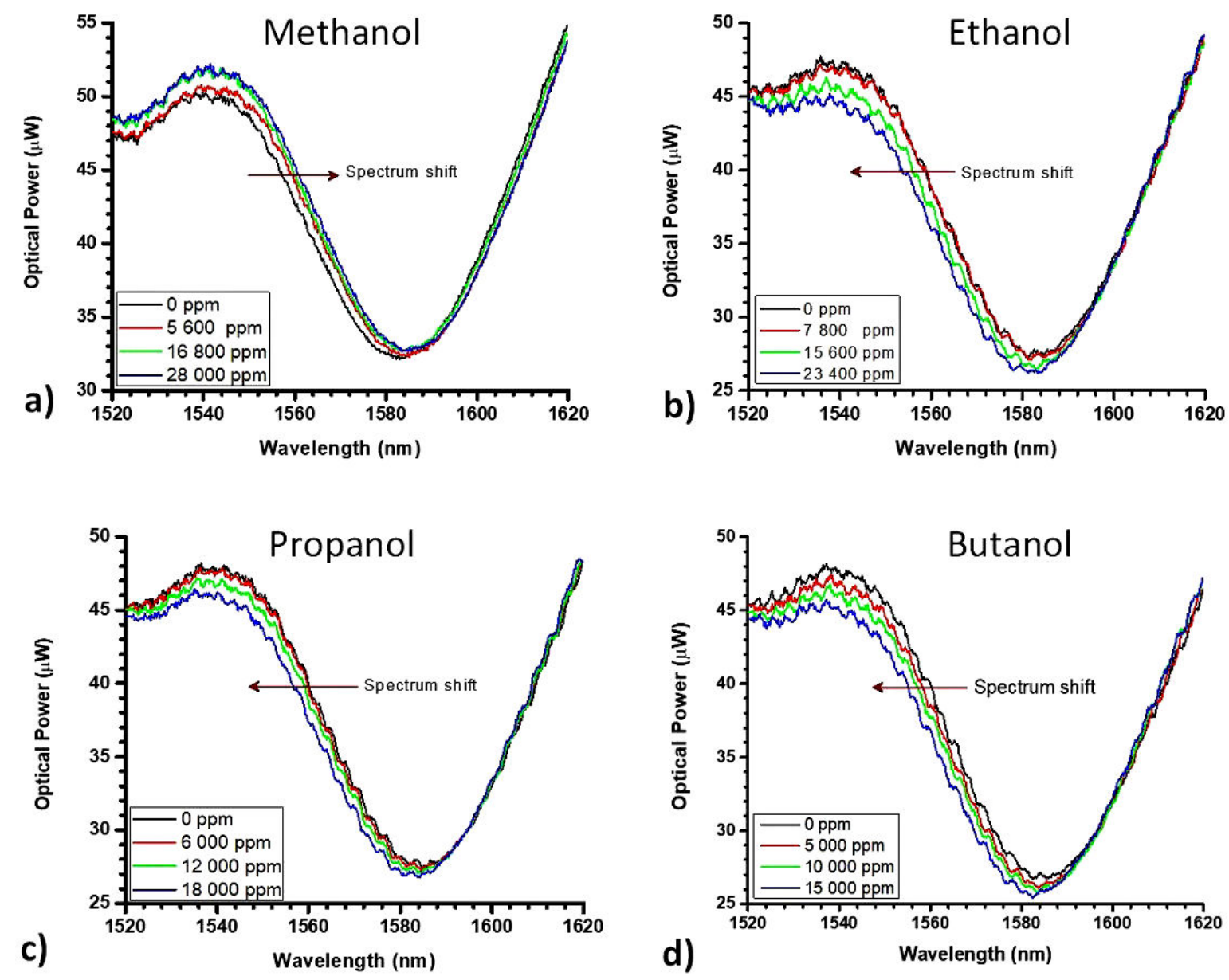

FIGURE 2. Transmission spectra at different sample concentrations for a) methanol, b) ethanol, c) propanol, and d) butanol. 

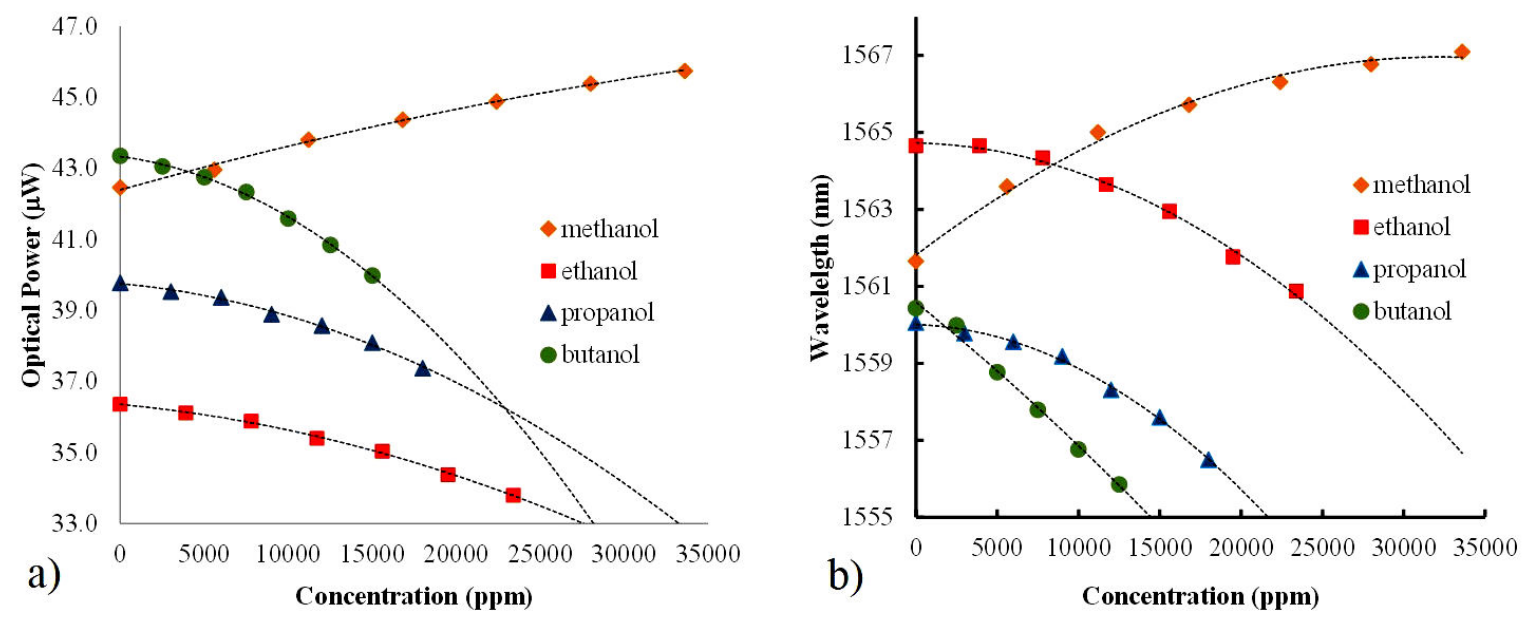

FIGURE 3. Calibration curves for the sensor response related to the sample concentration for a) optical power at a fixed wavelength of $1560 \mathrm{~nm}$ and b) for wavelength at a fixed optical power of $40 \mu \mathrm{W}$.

chamber, where the sensor was placed; and a power meter connected to the PC, where the data were stored for analysis. The alcohol liquid samples were injected with a micrometric syringe. The samples measured were methanol, ethanol, 1-propanol, and 1-butanol, which were injected in liquid amounts of $10 \mu \mathrm{l}$ at a time from 10 to $60 \mu \mathrm{l}$ for each one of the samples. For simplicity, the samples 1-propanol and 1-butanol will be addressed as propanol and butanol, respectively. The concentrations for these liquid amounts were calculated using the equation reported in previous work [17]. For this case, the calculated concentrations for $10 \mu \mathrm{l}$, were as follows, $5600 \mathrm{ppm}$ for methanol, $3900 \mathrm{ppm}$ for ethanol, $3000 \mathrm{ppm}$ for propanol, and $2500 \mathrm{ppm}$ for butanol. The measurements were performed at a constant room temperature (around $25^{\circ} \mathrm{C}$ ) with no temperature control. However, it is worth saying that there were no large temperature fluctuations.

LPFG are defined by a periodic variation of the core refractive index in an optical fiber. They couple core guided modes into forward propagating cladding modes. This characteristic is dependent on the wavelength. Therefore the LPFG transmission spectrum presents a minimum in a wavelength value determined by Eq. (1) [6],

$$
\lambda_{m}=\left(n_{c o}-n_{c l}^{m}\right) \Lambda
$$

where $\lambda_{m}$ is the resonance wavelength between the core propagating modes and the cladding propagating ones, $n_{c o}$ is the effective refractive index of the core, and $n_{c l}^{m}$ is the $m-t h$ mode in the cladding. $\Lambda$ is the period of the LPFG. In this equation, a variation of the effective refractive index of the cladding, which is directly affected by the sensing film, would produce a shift of the LPFG transmission spectrum. Therefore, the interaction of the sensing film with different organic vapors would provoke transmission spectrum changes.

\section{Results and discussion}

The transmission spectra measured for different concentrations are shown in Fig. 2 for all the samples: (a) methanol, (b) ethanol, (c) propanol, and (d) butanol. In all the cases, only three out of the six transmission spectra are shown to present the spectrum shifts more clearly. These spectra presented a variation of amplitude, especially in the wavelength range from 1520 to $1585 \mathrm{~nm}$. The latter value is precisely the peak of minimum transmission. There are no significant changes for wavelengths larger than $1585 \mathrm{~nm}$. The transmission variations are different for each sample; for instance, in the methanol case, transmission amplitude increment was observed, which occurs as a power increase when a constant wavelength is observed. On the other hand, when the power is constant, the spectrum changes can be appreciated as wavelength shifts towards larger values. In the other three sample cases (ethanol, propanol, and butanol), there is an opposite behavior, i.e., the optical power decreases as the sample concentration increases. This behavior is analyzed below.

From the curves displayed in Fig. 2, the results for the optical power variations related to the alcohol vapor concentration at a fixed wavelength value of $1560 \mathrm{~nm}$ were obtained. Figure 3a) shows these results. Similarly, the wavelength shifts according to the alcohol vapor concentration at a fixed optical power of $40 \mu \mathrm{W}$ are displayed in Fig. 3b). In the case of methanol, as mentioned above, the optical power increases as sample concentration does, whereas variations occur in the opposite direction in the other three types of alcohol; that is to say, there is a decrease in the optical power as sample concentration increases. Similar behavior is observed in wavelength shifts according to sample concentration. Therefore, it is possible to operate the sensor using two different variables, the optical power, and the wavelength. It is more convenient to operate the sensor with optical power since a single conventional photodetector can be used in this case, and the measurement system can be much simpler, whereas if the 

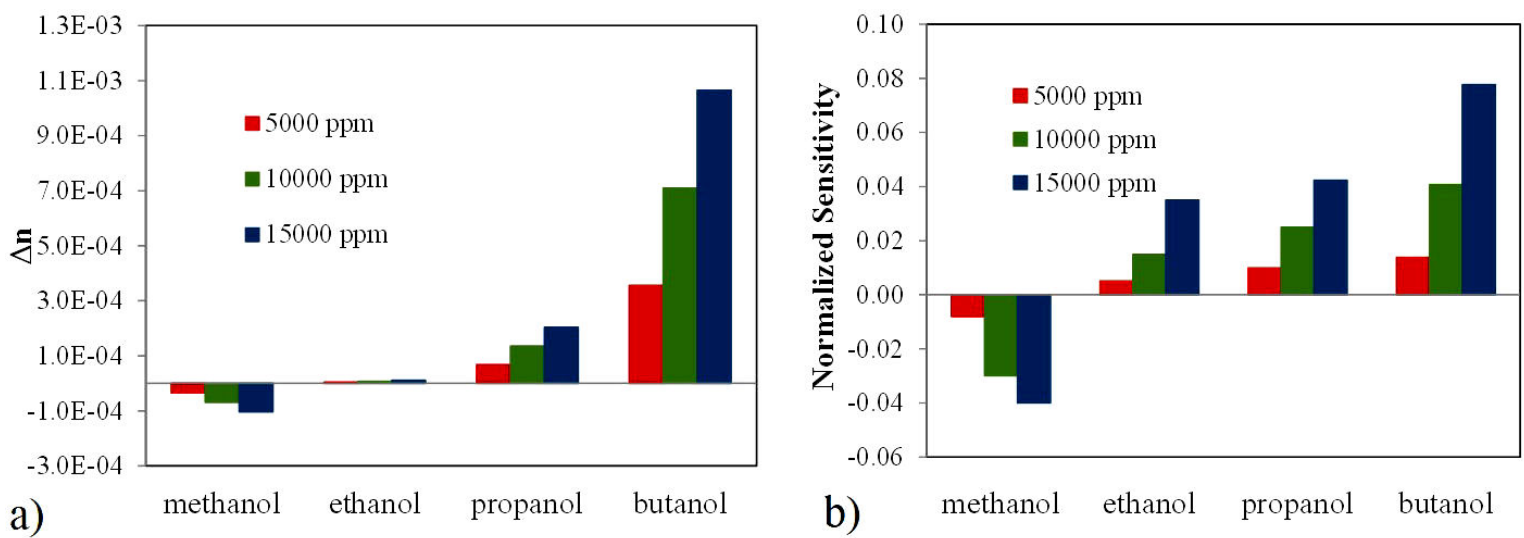

FIGURE 4. a) Refractive index variations due to the samples at concentrations of 5000, 10000 and 15000 ppm, and b) normalized sensitivity in optical power for the experimental data at the same sample concentrations as in a).

sensor is operated with wavelength, an optical spectrum analyzer (OSA) is required. On the other hand, curve fittings were performed, where the best fitting was found to be the one performed with a second-order polynomial. The correspondent correlation coefficients were above 0.9932 in the case of the optical power and larger than 0.9890 in the case of the wavelength. These values mean that the data dispersion is relatively small and, thus, it can be said that there is a well-defined relationship between the sensor response and the sample concentration. Since the sensor response is not linear, the sensitivity is not the same for any concentration value, but it can be calculated by the derivative of the response related with the sample concentration. The value obtained would depend on the sample measured since the concentration ranges are different.

One of the main characteristics of an LPFG is its sensitivity to refractive index variations. Therefore, the refractive index variation $(\Delta n)$ provoked by the interaction with alcohol molecules was calculated. Such calculation was performed using the concentration of the alcohol molecules inside the PDMS sensing film, which depends on the concentration of the measured sample in the air, and is given by the partition coefficient. This parameter is the relationship between the concentration inside the sensing film $\left(C_{f}\right)$ to that in the air $\left(C_{g}\right)$ and is given by Eq. (2) [17].

$$
K=\frac{C_{f}}{C_{g}} .
$$

The calculations were performed supposing a uniform distribution of the alcohol molecules in the PDMS sensing film and a linear refractive index variation of the mixture PDMS-Alcohol, which is a good approximation for low concentrations. In these calculations, the partition coefficients $(K)$ of the alcohols in PDMS were used. In this case, the $K$ value of methanol was estimated from the values in the other samples (ethanol, propanol, and butanol), which are reported in the literature [18]. Figure 4a) shows the calculated refractive index variation of the sensing film (PDMs) caused by its interaction with the same sample concentration. The $\Delta n$ changes are in the order of $10^{-4}$, and in the case of ethanol, such variations are tiny. This is due to the similarity of both refractive indexes (Ethanol and PDMS). On the other hand, Fig. $4 \mathrm{~b}$ ) shows the normalized sensitivity change of the optical power obtained from the data shown in Fig. 3a) for the same conditions as those mentioned in Fig. 4a). Similar behavior can be observed, a decrement in the sensor response for methanol and an increment for ethanol, propanol, and butanol. Furthermore, it can be observed that as the molecular weight of the sample increases, the sensitivity also increases since there are different refractive index variations for the same concentration. Consequently, it can be said that the change in the refractive index per concentration unit, which is related to the intensity variation, may provide information about the kind of sample being measured. Therefore, by using this characteristic, the sensor may classify the kind of sample under measurement according to its molecular weight. This is especially important when a VOC recognition is required to be performed using sensor arrays, since this feature can be used together with other sensor parameters such as steady-state sensor response, transient response, functional group, among others in order to increase the effectiveness of the array.

The sensor responses were studied using principal component analysis (PCA). PCA is a statistical tool to identify

TABLE I. Calibration curves for each sample in function of sample concentration (C).

\begin{tabular}{ccc}
\hline Sample & Calibration equation & $\begin{array}{c}\text { Correlation } \\
\text { coefficient }\left(R^{2}\right)\end{array}$ \\
\hline Methanol & $P C A 1=-1 \times 10^{-9} \mathrm{C}$ & 0.9512 \\
Ethanol & $P C A 1=5 \times 10^{-14} \mathrm{C}^{2}$ & \\
& $+4 \times 10^{-10} \mathrm{C}$ & 0.9813 \\
Propanol & $P C A 1=6 \times 10^{-14} \mathrm{C}^{2}$ & \\
& $+5 \times 10^{-10} \mathrm{C}$ & 0.9875 \\
Butanol & $P C A 1=3 \times 10^{-9} \mathrm{C}$ & 0.9882 \\
\hline
\end{tabular}




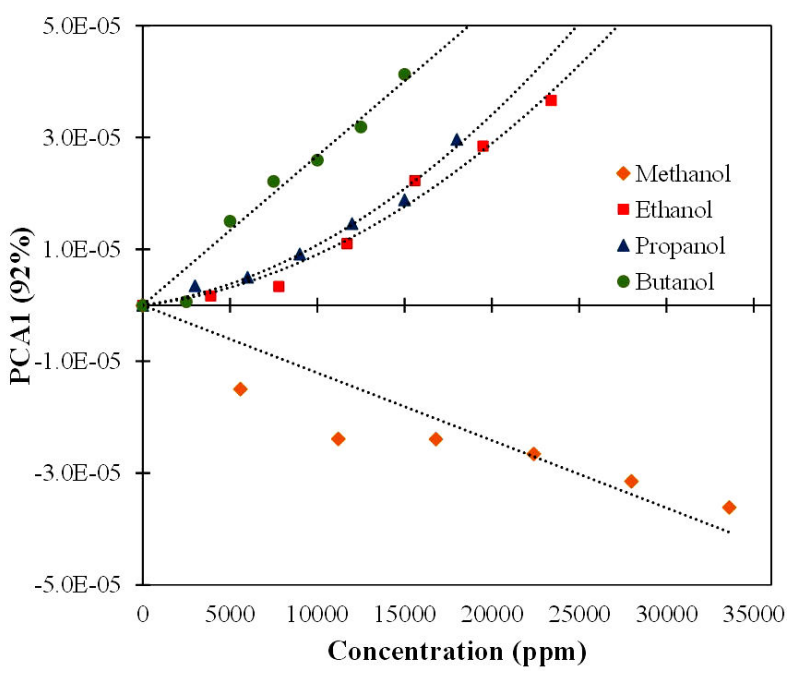

FIGURE 5. PCA results for the four LPFG sensors.

patterns and shapes and can be used in spectral analysis. The advantage of using PCA is that each data point corresponds to a complete LPFG spectrum measured for a specific sample concentration. This increases the robustness of the sensor characterization since it is equivalent to perform the calibration curves using an array of 400 photodetectors (400 data points in the spectrum for the case of this work), all of them located $0.5 \mathrm{~nm}$ from each other in wavelength. PCA results are shown in Fig. 5. We plotted PC1 vs. sample concentration. We used the variance-covariance matrix to perform the PCA. The variance contained in PC1 was $92 \%$, which means a high information percentage displayed in this principal component. Notice that all the curves are referenced to 0 since the initial spectrum was measured at $0 \mathrm{ppm}$. We observe that the curve for methanol has a different behavior than that of the other samples (ethanol, propanol, and butanol), it moves towards negative values. This is in agreement with the behavior shown in Figs. 2, where the LPFG spectrum for methanol shifts to the right, while for the other samples the spectrum shifts to the left. We think that such behavior is due to the refractive index variation of the mixture PDMS-alcohol sample. Moreover, the calibration curves for the trendlines shown in Fig. 5 are shown in Table I, together with their correlation coefficients $\left(R^{2}\right)$. Methanol and butanol have a linear behavior and ethanol and propanol have a second-order polynomial. The correlation coefficients are above 0.9512 . For comparison purposes, we can mention that the resolution of the ethanol sensor is around $1400 \mathrm{ppm}$, which is quite similar to the result obtained in a previous work of our group, where a value of $1500 \mathrm{ppm}$ was obtained using PDMS [11]. We can see that the curves for ethanol and propanol are quite similar and for some applications, these two samples may not be discriminated against. However, the PCA method can be combined with some other sensors through sensor arrays to be able to discriminate between these two samples.

\section{Conclusion}

In this work, an alcohol sensor was developed using a PDMS sensing film deposited on an LPFG by the immersion technique. The sensor was able to detect the VOCs, particularly alcohols, through the adsorption of sample molecules into the sensing film. This VOC detection produced refractive index variations in the sensing film, which, in turn, generated changes in the transmission profile of the LPFG. The changes in the transmission profile can be interpreted as wavelength shifts when a constant optical power is fixed or as optical power variations when a wavelength is constant. The sensor response exhibited a behavior that fitted adequately to a second-order polynomial relation to the sample concentration with correlation coefficients above 0.9932 in the case of the optical power and 0.9890 in the case of the wavelength. PCA results showed a similar behavior to the other analysis, with the advantage that is not necessary to use a particular wavelength, but the complete LPFG spectrum. The calibration curves were determined and correlation coefficients above 0.9512 were found. Furthermore, the sensor response curves were found to reveal a different behavior for methanol, compared with the other samples (ethanol, propanol, and butanol) for all cases, wavelength shifts, spectral amplitude variations, and PC1 changes. This behavior may be attributed to the fact that the refractive index of methanol is less than the one of PDMS, whereas the values of the other samples are larger. Therefore, the effects on the adsorption of the alcohol vapor molecules must be different.

\section{Acknowledgments}

This work was partially supported by the project SEPCONACYT, CIENCIA BÁSICA-2016, grant number 286647.
1. S. Muñoz-Aguirre, A. Yoshino, T. Nakamoto and T. Moriizumi, Odor Approximation of Fruit Flavors Using a QCM Odor Sensing System, Sens. Actuat. B, 123 (2007) 1101. https: //doi.org/10.1016/j.snb.2006.11.025

2. J. W. Gardner and J. Yinon, Electronic Noses and Sensors for the Detection of Explosives, (Ed. Kluwer Academic Publishers, 2004).
3. J. W. Gardner and P. N. Bartlett, Electronic Noses, (Oxford University Press, 1999).

4. S. Jiang, and Y. Liu, Gas sensors for volatile compounds analysis in muscle foods: A review, Trends in Analytical Chemistry 126 (2020) 115877. https://doi.org/10.1016/ j.trac.2020.115877

5. J. Zhou, Zi-Ao Huang, U. Kumar, and D. D. Y. Chen, Review 
of recent developments in determining volatile organic compounds in exhaled breath as biomarkers for lung cancer diagnosis, Analytica Chimica Acta 996 (2017) 1. http: / / dx . doi. org/10.1016/j.aca.2017.09.021

6. H.J. Patrick, A.D. Kersey, F. Bucholtz, Analysis of the response of long period fiber gratings to external index of refraction, J. Light. Technol. 16 (1998) 1606. https : //doi .org/10. $1109 / 50.712243$

7. J. Hromadka, B. Tokay, S. James, R.P. Tatam, and S. Korposh, Optical fibre long period grating gas sensor modified with metal organic framework thin films, Sensors Actuators, B Chem. 221 (2015) 891. https://doi.org/10.1016/ j.snb.2015.07.027

8. J. Hromadka, S. Korposh, and M. Partridge, Volatile Organic Compound Sensing Using Optical Fiber Long-Period Grating with mesoporous nano-scale coating, Sensors, 17 (2017) 1. https://doi.org/10.3390/s17020205

9. M. Singh, S.K. Raghuwanshi, and O. Prakash, UltraSensitive Fiber Optic Gas Sensor Using Graphene Oxide Coated Long Period Gratings, IEEE Photonics Technol. Lett. 31 (2019) 1473.. https : / / doi .org/10.1109/ LPT.2019.2932764

10. J. Barnes, M. Dreher, K. Plett, R. S. Brown, C. M. Crudden, and H. P. Loock, Chemical sensor based on a long-period fibre grating modified by a functionalized polydimethylsiloxane coating. Analyst, 133 (2008) 1541. https://doi:10.1039/ b806129g

11. C. Martínez-Hipatl, S. Muñoz-Aguirre, G. Beltrán-Pérez, J. Castillo-Mixcóatl and J. Rivera de la Rosa, Detection of volatile organic compounds by an interferometric sensor, Sens. Actuat. B, 147 (2010) 37.https://doi.org/10.1016/j.snb. 2010.03 .042
12. R. Cao et al., Metal-organic framework functionalized polymer coating for fiber optical methane sensors, Sens and Actuat: B. Chemical, 324 (2020) 1. https://doi.org/10. $1016 / j . s n b .2020 .128627$

13. G. L. Klunder and R. E. Russo, Core Based Intrinsic Fiber Optic Absorption Sensor for the Detection of Volatile Organic Compounds, Applied Spectroscopy 49 (1995) 379. https: //doi.org/10.1366/0003702953963580

14. J.A. Barnes, R.S. Brown, A.H. Cheung, M.A. Dreher, G. Mackey and H. P. Loock, Chemical Sensing Using a Polymer Coated Long Period Fiber Grating Interrogated by RingDown Spectroscopy, Sens. Actuat. B 148 (2010) 221. https: //doi.org/10.1016/j.snb.2010.04.007

15. Jian Zhang, Xiling Tang, Junhang Dong, Tao Wei and Hai Xiao, Zeolite Thin Film Coated Long-Period Fiber Grating Sensor for Measuring Trace Organic Vapors, Sens. Actuat. B 135 (2009) 420. https://doi.org/10.1016/j.snb. 2008.09 .033

16. S. Y. Tan, Y. T. Yong, S. C. Lee, and F. Abd Rahman, Review on an arc-induced long-period fiber grating and its sensor applications. Journal of Electromagnetic Waves and Applications, 29 (2015) 703. https://dx.doi.org/10.1080/ 09205071.2015 .1021019

17. M. Rodríguez-Torres, V. Altuzar, C. Mendoza-Barrera, G. Beltrán-Pérez, J. Castillo-Mixcóatl and S. Muñoz-Aguirre, Discrimination Improvement of a Gas Sensors' Array Using HighFrequency Quartz Crystal Microbalance Coated with Polymeric Films, Sensors 20 (2020) 6972, https : //doi.org/ $10.3390 / \mathrm{s} 20236972$

18. A. Hierlemann, E.T. Zellers, A.J. Ricco, Use of linear solvation energy relationships for modeling responses from polymercoated acoustic-wave vapor sensors, Anal. Chem. 73 (2001) 3458. https://doi.org/10.1021/ac010083h 